\title{
Copy Move Forgery Detection Through Differential Excitation Component- Based Texture Features
}

\author{
Gulivindala Suresh, GMR Institute of Technology, India \\ iD https://orcid.org/0000-0002-8411-8190 \\ Chanamallu Srinivasa Rao, Jawaharlal Nehru Technological University, Kakinada, India
}

\begin{abstract}
Copy-move forgery (CMF) is an established process to copy an image segment and pastes it within the same image to hide or duplicate a portion of the image. Several CMF detection techniques are available; however, better detection accuracy with low feature vector is always substantial. For this, differential excitation component (DEC) of Weber Law descriptor in combination with the gray level co-occurrence matrix (GLCM) approach of texture feature extraction for CMFD is proposed. GLCM Texture features are computed in four directions on DEC and this acts as a feature vector for support vector machine classifier. These texture features are more distinguishable and it is validated through other two proposed methods based on discrete wavelet transform-GLCM (DWT-GLCM) and GLCM. Experimentation is carried out on $\mathrm{CoMoFoD}$ and CASIA databases to validate the efficacy of proposed methods. Proposed methods exhibit resilience against many post-processing attacks. Comparative analysis with existing methods shows the superiority of the proposed method (DEC-GLCM) with regard to detection accuracy.
\end{abstract}

\section{KEYWORDS}

Copy Move, Differential Excitation Component, GLCM, Image Forgery, Support Vector Machine, Weber Law Descriptor

\section{INTRODUCTION}

Digital images are being edited deliberately or involuntarily to make them more informative or to hide some content in the image. The vast growth of commercial and open source digital photo editing tools leads to the increase of tampered images in day-to-day life. The trustworthiness of digital image plays a major role in many applications, viz., criminal examination, journalism, forensic analysis and surveillance systems (Mahdian \& Saic, 2010). A beginner in digital forensics area can refer to its various applications in (Li, 2013). Digital Image Forgery (DIF) detection is plausible in two approaches (Hashmi \& Keskar, 2015), viz., Active and Passive. Active approach involves pre-processing of a genuine image by embedding an identifier before it is used. Watermarking and signature embedding technologies are active approaches useful for detection and localization of image forgery but pre-

This article, originally published under IGI Global's copyright on July 1, 2020 will proceed with publication as an Open Access article starting on January 27, 2021 in the gold Open Access journal, International Journal of Digital Crime and Forensics (converted to gold Open Access January 1, 2021), and will be distributed under the terms of the Creative Commons Attribution License (http://creativecommons.org/ licenses/by/4.0/) which permits unrestricted use, distribution, and production in any medium, provided the author of the original work and original publication source are properly credited. 
processing of digital data limit its usage. Passive method (Al-Qershi \& Khoo, 2013) explore statistics or features from an image for CMFD.

Copy-Move type of forgery is one in which some snippet of an authentic image is copied and pasted within the image with an intent to hide a specific content in the image. The pasted portion relates to authentic image, hence it affects statistical properties of the image and these variations are explored to detect the forgery. It is clear from Figure 1, that the forgery does not leave any visual

Figure 1. Copy-move forgery: (a) CMF image; (b) Original image

a. Copy Move Forgery

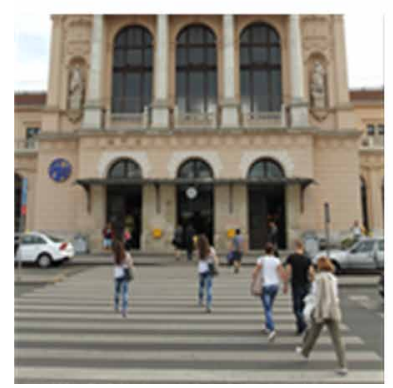

b. Original Image

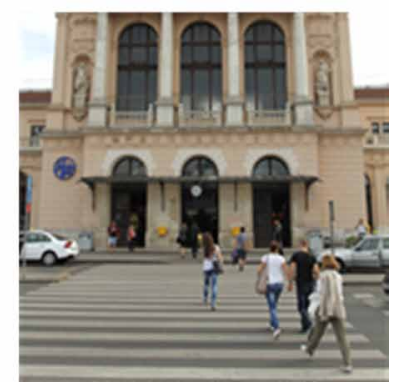

clue to identify the tampering. CMF problem can be addressed in two ways, i. Localization and ii. Detection. The localization process recognizes at which locations the image is being tampered whereas the detection process classifies whether the given image is forged or not.

The CMF localization process is shown in Figure 2 and it focusses on extracting the features from the overlapping blocks of the suspicious image in the block-based methods. In the case of key-point

Figure 2. CMF localization process

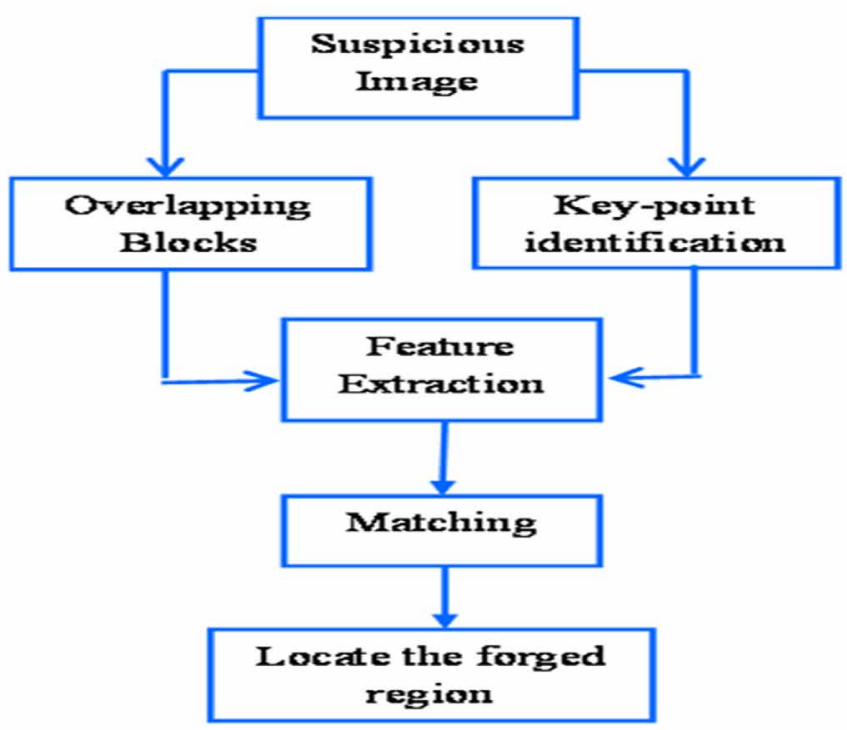


based methods, it explores the key-points, i.e. high-entropy regions of the image. Feature matching is performed to identify similar blocks or key-points in the given image. These matched regions are considered as potential blocks of forgery.

The CMF detection process is illustrated in Figure 3. The detection process involves extracting features from all the images in the dataset. A suspicious image is tested against the trained set to confirm whether it is an original or forged image.

Figure 3. CMF detection process

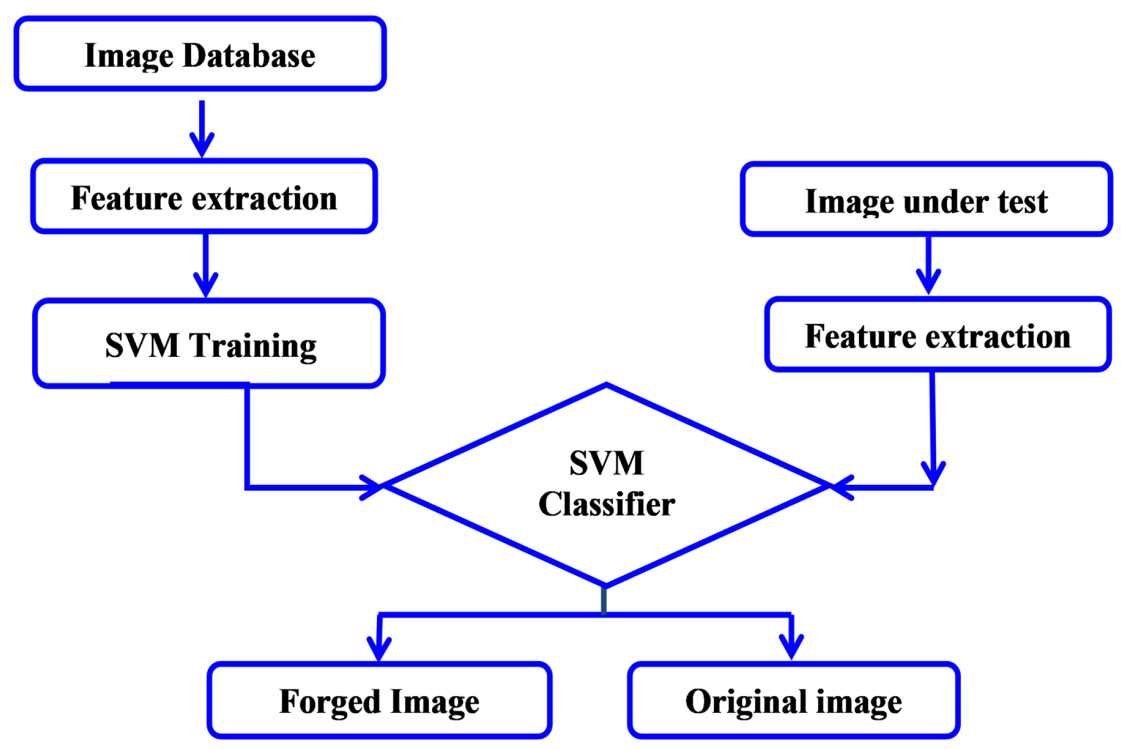

The detection process seems to be uncomplicated from Figure 3 and it is true for simple copy move forgeries. However, CMF images can be affected by various post-processing attacks viz., JPEG compression, blurring, noise addition, and color reduction, etc. Detecting the images under these attacks is critical and several methods are available in the literature which can identify simple CMF images. Authors in (Shen et al., 2017) developed an image splicing detection method using textural features from Gray Level Co-occurrence Matrix (GLCM) but it has not concentrated on CMFD.

Another work developed by (Suresh \& Srinivasa Rao, 2016) based on GLCM texture features for CMFD is available. It needs 22 statistical features and these are calculated in one direction. Even though other methods are able to handle complicated forgeries, but they are computationally expensive. Moreover, the feature set of an image plays a critical role in the classification.

In order to address these challenges, in this paper, three methods are proposed for CMFD. In proposed method-1 (GLCM), six GLCM textural features are computed in four directions to yield 24 features for classification. The proposed method-2 is based on Discrete Wavelet Transform (DWT) and GLCM. DWT is applied on the image and GLCM textural features are computed on low frequency sub-band of the image. In proposed method-3, a novel combination of Differential Excitation Component with GLCM (DEC-GLCM) is explored to extract texture features for CMFD. These texture features are more distinguishable and this is validated through two proposed methods based on i. DWT-GLCM, ii. GLCM and other existing methods. Also, the feature size of the three proposed methods is low.

Rest of the paper is structured as follows. A detailed review of various CMFD methods is discussed in Section 2. The feature extraction using DEC-GLCM and DWT-GLCM is presented in Section 3. 
The three proposed methods based on GLCMs are elucidated in Section 4. Section 5 focuses on the validation of proposed methods with results and comparative analysis of the proposed methods with existing methods. Concluding remarks are presented in Section 6.

\section{RELATED WORK}

CMFD became a trending research area and many researchers have contributed techniques to address CMF detection and localization. A methodological review of CMFD techniques is being presented by Sekhar and Shaji (2014) and Dixit and Naskar (2017). The initial work on CMFD was reported by Fridrich et al. (Diane, 2003) using DCT coefficients for feature extraction. But the technique cannot withstand additive noise. CMFD technique using Principal Component Analysis (PCA) was proposed by Popescu et al. (Popescu \& Farid, 2004) which has resilience against additive noise but with low detection accuracy. Several works on CMF detection and localization are available in the literature, but CMF detection techniques are reviewed here as the proposed work is related to detection approach.

Weber Law Descriptors (WLD) are explored by (Hussain et al., 2012) on chrominance components for CMFD. Robust texture features are built from WLDs at different scales. The method is experimented for different types of copied regions. A CMFD method based on Curvelet and Local Binary Pattern (LBP) approach is used by (Al-Hammadi et al., 2013) for the classification of forged images. LBP is applied to the sub-bands obtained from Curvelet transform at different scales and orientations. The final histogram is formed from the fusion of normalized LBP histograms and this act as a feature vector. This method is evaluated on the CASIA database and achieved an accuracy of $93.4 \%$. Steerable Pyramid Transform (SPT) and LBP are used by (Muhammad et al., 2013) for CMFD. LBP is applied to the sub-bands obtained from SPT at different scales and orientations. Another work with SPT-LBP is proposed by (Muhammad et al., 2014) and here, feature selection methods are explored to reduce the dimensionality of the feature vector. The method is evaluated with the features from different Chroma components. Multi-scale LBP is explored with Multi-scale WLD by (Hussain et al., 2015) for CMFD. Robust texture features are developed with WLD and LBP at different scales and this method has been evaluated on the CASIA database.

Gabor wavelets and Local Phase Quantization (LPQ) are used by Isaac and Wilscy (2015) for forgery detection. LPQ is applied on sub-bands obtained from Gabor wavelets at different scales and orientations. Authors Hashmir and Keskar (2015) explored Discrete Cosine Transform (DCT), LBP, Curvelet, and Gabor for feature extraction. These features are trained with Hidden Markov Model (HMM) and Support Vector Machine (SVM) to classify the forged images from the original images. A CMFD method (Agarwal \& Chand, 2015) is proposed by Saurabh and Satish with entropy filter and LPQ on different color channels but it works with a large feature size of 2048. Authors (Mangat \& Kaur, 2016) used SIFT and Kernel Principal Component Analysis (KPCA) for CMFD. They have investigated SVM and Neural networks for classification. LBP texture features are used by (Alhussein, 2016) and these features are fed to Extreme Learning Machine (ELM) for classification. Agarwal and Chand (2017) extracted image features in the Undecimated DWT domain and Markov model is used for classification. Wu et al. (2017) explored DCT and two-scale LBP for feature extraction. This method is an image-format independent approach which can detect different type of tampered images. SVM and neural networks are used for classification. Vidyadharan and Thampi (2017) developed a CMFD based on multi-texture description using LBP, LPQ, Binary Gabor Pattern (BGP) and orientation using SPT. In this work, ReleifF algorithm is used for feature selection and random forest classifier is explored for classification. It has been evaluated on CASIA dataset. An integrated system is being developed by Prakash et al. (2018) for CMFD and splicing detection. This method used DCT for feature extraction through enhanced threshold method. Deep learning methods are also explored for CMFD, in (Rao \& Ni, 2016), a trained Convolutional Neural Network (CNN) is explored to extract dense features and SVM is used for classification. Similarly, Zhou and Rao (2017), used CNN with 
a novel blocking strategy for CMFD. These two deep learning techniques are basically data-driven approaches and provide an accuracy of $97 \%$ approximately.

The state-of-art methods reviewed here basically work with texture features in combination with a transform. Choice of image transforms along with other texture feature is being explored by researchers to reduce the feature vector size. The need of the hour is high detection accuracy with low feature vector or computational effort. Hence, in the proposed method, DEC of WLD Descriptor is used in combination with GLCM for texture feature extraction to build a more distinguishable feature vector.

Main contributions of the proposed work are 1. DEC is explored to compute local salient patterns in the image without considering orientation component which does not give local spatial information of the image. 2. DEC is combined with GLCM in four orientations to represent texture with reference to directions in order to handle the rotation attack. 3. Texture features (DEC-GLCM) are computed from the above representation and are used for CMFD. 4. Significance of usage of DEC with GLCM is validated through DWT-GLCM and GLCM based methods with regard to the accuracy, sensitivity, and specificity.

\section{MATERIALS AND METHODS}

In this section, DEC, DWT, GLCM and novel combination of DEC with GLCM for feature extraction and its significance is discussed as well its superiority to GLCM on DWT approximation sub-band is also presented.

\subsection{Differential Excitation Component}

WLD is a powerful local texture descriptor (Jie et al., 2009) comprises of two components: DEC and Orientation. DEC is the ratio of change in intensity between its neighbors to a current pixel. It gives local significant patterns in the image. DEC is computed as given in (1), firstly, change in intensity between its neighbors and the center pixel are calculated with filter $f_{00}$ and secondly, the ratio of the change in intensity of the current pixel by the outputs of the two filters $f_{00}$ and $f_{01}$. Figure 4 defines two filters $f_{00}$ and $f_{01}$.

Figure 4. Filters to obtain $\mathbf{v}_{\mathbf{s}}^{00}$ and $\mathbf{v}_{\mathbf{s}}^{01}:$ (a) Filter $\mathbf{f}_{00} ;$ (b) Filter $\mathbf{f}_{01}$

\begin{tabular}{|l|l|l|}
\hline+1 & +1 & +1 \\
\hline+1 & -8 & +1 \\
\hline+1 & +1 & +1 \\
\hline
\end{tabular}

\begin{tabular}{|c|c|c|}
\hline 0 & 0 & 0 \\
\hline 0 & +1 & 0 \\
\hline 0 & 0 & 0 \\
\hline
\end{tabular}

$v_{s}^{00}=\sum_{i=0}^{n-1}\left(P_{i}-P_{c}\right)$

where, $P_{i}(i=0,1, \ldots n-1)$ represents $i^{\text {th }}$ neighbors of $P_{c}$ and $n$ is available neighbors.

The ratio of the intensity differences obtained from filter $f_{00}$ to the current pixel obtained from filter $f_{01}$ is defined in (2): 
$I_{\text {ratio }}\left(P_{c}\right)=\frac{v_{s}^{00}}{v_{s}^{01}}$

Employing arctan function on $I_{\text {ratio }}(\cdot)$ :

$\arctan \left[I_{\text {ratio }}\left(P_{c}\right)\right]$

Combining Equations (1), (2) and (3), we have (4):

$\arctan \left[I_{\text {ratio }}\left(P_{c}\right)\right]=\arctan \left[\frac{v_{s}^{00}}{v_{s}^{01}}\right]$, therefore $\arctan \left[I_{\text {ratio }}\left(P_{c}\right)\right]=\arctan \left[\frac{\sum_{i=0}^{n-1}\left(P_{i}-P_{c}\right)}{P_{c}}\right]$

Hence, DEC of the current pixel $\zeta\left(P_{c}\right)$ is computed as given in (5):

$\zeta\left(P_{c}\right)=\arctan \left[\frac{v_{s}^{00}}{v_{s}^{01}}\right]=\arctan \left[\frac{\sum_{i=0}^{n-1}\left(P_{i}-P_{c}\right)}{P_{c}}\right]$

$\zeta\left(P_{c}\right)=\left\{\begin{array}{l}+v e, \quad \text { when neighbor pixels are brighter than center pixel } P_{c} \\ -v e, \quad \text { when neighbor pixels are darker than center pixel } P_{c}\end{array}\right.$

The input image and its DEC scaled to 8-bit gray level are illustrated in Figure 5.

Four GLCMs are obtained from image DEC to calculate the 24 texture feature vector as described in the next sub-sections.

\subsection{DWT}

DWT decomposes image at various levels to localize the signal both in time and frequency domains. This property leads to several applications like data compression, denoising, and image

Figure 5. Input image and it's differential excited component; a. input image; b. differential excitation component
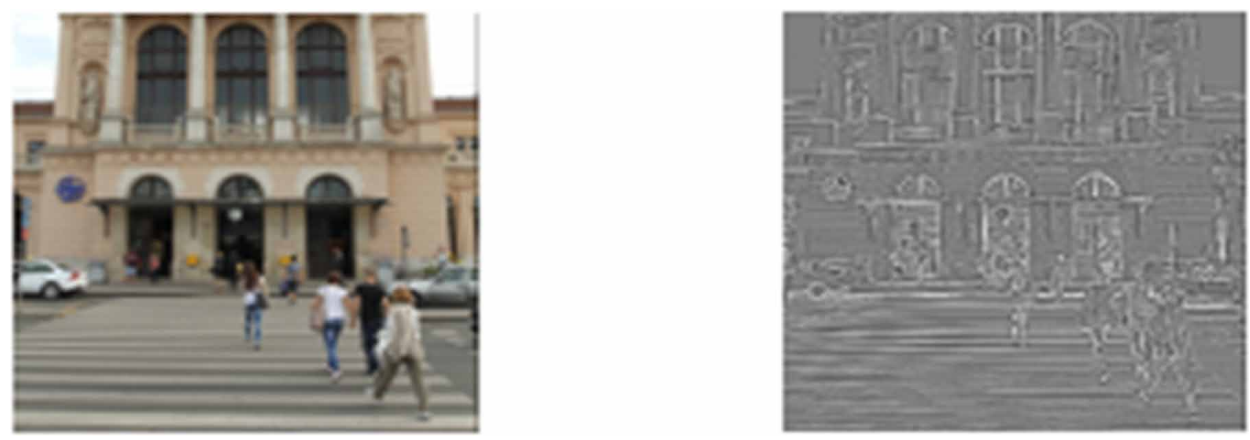
feature extraction and so on (Yang et al., 2013). The decomposed image consists of approximation and detail coefficients (such as horizontal, vertical and diagonal). In our proposed method DWTGLCM, approximation band of the single level decomposed image is considered which reduces the computational effort by $3 / 4$. GLCM is applied on DWT approximation band to compute the 24 texture features as described in the next sub-sections.

\subsection{GLCM}

GLCM is a good old technique to extract texture features and recently, it is used for applications viz., satellite images (Zhang et al., 2017) and forgery detection (Shen et al., 2017). Texture features are computed from GLCM as given by Haralick et al. (1973). GLCM tabulates co-occurrence of various combinations of gray intensities in an image segment. It is useful to extract second order textural features from gray images. Co-occurrence of gray levels is calculated using two parameters, relative distance of the pixel pair $d$ and their relative orientation $\theta$. Four relative orientations for $\theta$ are considered $\left\{\theta=0^{\circ}, \theta=45^{\circ}, \theta=90^{\circ}, \theta=135^{\circ}\right\}$ to yield GLCMs G4 $=\{\mathrm{G}(0,1), \mathrm{G}(-1,1), \mathrm{G}(-1,0)$ and $\mathrm{G}(-$ $1,-1)\}$ respectively and are shown in Figure 6.

Figure 6. Co-occurrence matrix directions for feature extraction

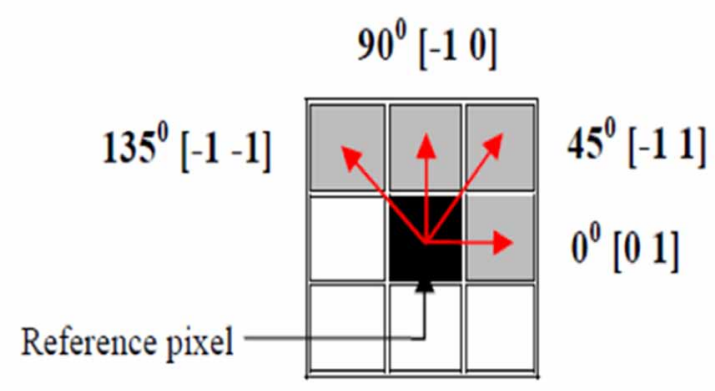

\subsection{Feature Extraction}

GLCM is constructed in three approaches for the three proposed methods and is respectively as follows, 1. from input gray image, 2. from DWT approximation sub-band and 3. from DEC of the input image. The input RGB image is transformed to intensity image and DEC of the image is obtained. DEC provides smooth as well as the high-frequency content of the image whereas the DWT approximation band provides low-frequency content of the image. GLCMs provide a histogram of gray intensities of different combinations, however further analysis is required to compute numeric features from GLCMs for the effective representation of texture.

Table 1 shows the list of 6 statistical features derived from a normalized GLCM. Texture features from GLCM in all four directions are computed on DEC image and this method of feature extraction is referred as DEC-GLCM in our proposed work and similarly, features extracted from GLCMs on DWT approximation band are called as DWT-GLCM.

These set of 6 textural features $F 6=\left\{F_{1}, F_{2}, F_{3}, F_{4}, F_{5}, F_{6}\right\}$ are extracted from each GLCM and is repeated for all GLCMs in set G4 to yield 24 texture features. These 24 textural features are used as a feature vector to verify the authenticity of the image. Figure 7 illustrates the DEC and DWT-approximation details of authentic and forged images. 
Table 1. GLCM texture feature description

\begin{tabular}{|c|c|c|}
\hline Textural Features & Equations & Feature Description \\
\hline $\begin{array}{l}\text { Angular } \\
\text { Secondary } \\
\text { Moment }\end{array}$ & $F_{1}=\sum_{i=1}^{n} \sum_{j=1}^{n} p^{2}(i, j)$ & $\mathrm{F}_{1}$ measures textural uniformity \\
\hline Entropy & $F_{2}=\sum_{i=1}^{n} \sum_{j=1}^{n} p(i, j) \log (p(i, j))$ & $\begin{array}{l}\mathrm{F}_{2} \text { defines the non-uniformity in an image. } \\
\text { Texturally non-uniform image segments result } \\
\text { in small entropy. }\end{array}$ \\
\hline Contrast & $F_{3}=\sum_{i=1}^{n} \sum_{j=1}^{n}(i-j)^{2} p(i, j)$ & $\begin{array}{l}\mathrm{F}_{3} \text { gives the contrast of the image. Higher } \\
\text { contrast provides a clearer image whereas } \\
\text { lower contrast results in a fuzzier image. }\end{array}$ \\
\hline Correlation & $\begin{array}{l}F_{4}=\frac{\sum_{i=1}^{n} \sum_{j=1}^{n}[(i, j) p(i, j)]-\mu_{1} \mu_{2}}{\sigma_{1} \sigma_{2}} \\
\text { Where, } \\
\mu_{1}=\sum_{i=1}^{n} i \sum_{j=1}^{n} p(i, j) \\
\mu_{2}=\sum_{j=1}^{n} j \sum_{i=1}^{n} p(i, j) \\
\sigma_{1}=\sum_{i=1}^{n}\left(i-\mu_{1}\right) \sum_{j=1}^{n} p(i, j) \\
\sigma_{2}=\sum_{j=1}^{n}\left(j-\mu_{2}\right) \sum_{i=1}^{n} p(i, j)\end{array}$ & $\begin{array}{l}\mathrm{F}_{4} \text { provides gray level dependencies in the } \\
\text { image. Higher correlation indicates that the } \\
\text { GLCM elements are uniform. }\end{array}$ \\
\hline Inverse Moment & for & $\begin{array}{l}\mathrm{F}_{5} \text { measures the degree of changes in the local } \\
\text { image texture. }\end{array}$ \\
\hline $\begin{array}{l}\text { Inverse Difference } \\
\text { Moment }\end{array}$ & $F_{6}=\sum_{i=1}^{n} \sum_{j=1}^{n} \frac{1}{1+(i-j)^{2}} p(i, j)$ & $\begin{array}{l}\mathrm{F}_{6} \text { measures image homogeneity. Smaller } \\
\text { GLCM elements result in larger } \mathrm{F}_{6} .\end{array}$ \\
\hline
\end{tabular}

\section{PROPOSED METHODS}

CMFD methods' perform the detection by classification of forged from the original images. To achieve this, features are extracted for all the original and forged images in the database. An SVM is trained with these feature vectors and a model is developed. This SVM model is used for classification. A novel CMFD using texture features obtained from GLCM on DEC is proposed and significance of texture features obtained from GLCM and DWT-GLCM is also presented. Here, three algorithms (algorithm 1-3) are developed and all the proposed methods 1. GLCM, 2. DWT-GLCM and 3. DECGLCM are presented as follows: 


\footnotetext{
Algorithm 1. Proposed method-1: CMFD using GLCM features

Input: Image Dataset
}

Output: Detection as Authentic or Forged

For each Image do

1. Convert color image to gray image

2. Obtain GLCM in all four directions i.e. $\left\{\theta=0^{\circ}, \theta=45^{\circ}, \theta=90^{\circ}, \theta=135^{\circ}\right\}$ to yield four GLCMs

3. Compute six statistical features $\left\{F_{1}, F_{2}, F_{3}, F_{4}, F_{5}, F_{6}\right\}$ on each GLCM to yield 24-dimension texture features vector

4. Train SVM Model using polynomial kernel

5. Test the trained SVM model to distinguish the original image from forged images

End For

Algorithm 2. Proposed method-2: CMFD using DWT-GLCM features

Input: Image Dataset

Output: Classified as Authentic or Forged

For each Image do

1. Convert color image to gray image

2. Apply DWT on gray image to obtain LL, LH, HL and HH bands

3. Construct GLCM on LL band in all four directions i.e. $\left\{\theta=0^{\circ}, \theta=45^{\circ}, \theta=90^{\circ}, \theta=135^{\circ}\right\}$ to obtain four GLCMs

4. Compute six statistical features $\left\{F_{1}, F_{2}, F_{3}, F_{4}, F_{5}, F_{6}\right\}$ on each GLCM to yield 24-dimension texture feature vector

5. Train SVM Model using polynomial kernel

6. Test the trained SVM model to identify the image as original or forged.

End for

Algorithm 3. Proposed method-3: CMFD using DEC- GLCM features

Input: Image Dataset

Output: Classified as Authentic or Forged

For each Image do

1. Convert color image to gray image

2. Obtain DEC of gray image

3. Construct GLCM on DEC in all four directions i.e. $\left\{\theta=0^{\circ}, \theta=45^{\circ}, \theta=90^{\circ}, \theta=135^{\circ}\right\}$ to construct four GLCMs

4. Compute six statistical features $\left\{F_{1}, F_{2}, F_{3}, F_{4}, F_{5}, F_{6}\right\}$ on each GLCM to yield 24-dimension texture feature vector

5. Train SVM Model using polynomial kernel

6. Test the trained SVM model to classify the image as authentic or forged.

End for

\section{EXPERIMENTAL RESULTS AND ANALYSIS}

This section describes the experimentation and validation of the three proposed methods GLCM, DWTGLCM, and DEC-GLCM. Initially, image dataset and SVM classification are elucidated, followed by the discussion on experimentation. Three sets of experiments are carried out to validate the proposed methods. The first set of experiments is used to show the performance of the proposed methods. The 
Figure 7. Illustration of DEC and DWT-Approximation band content

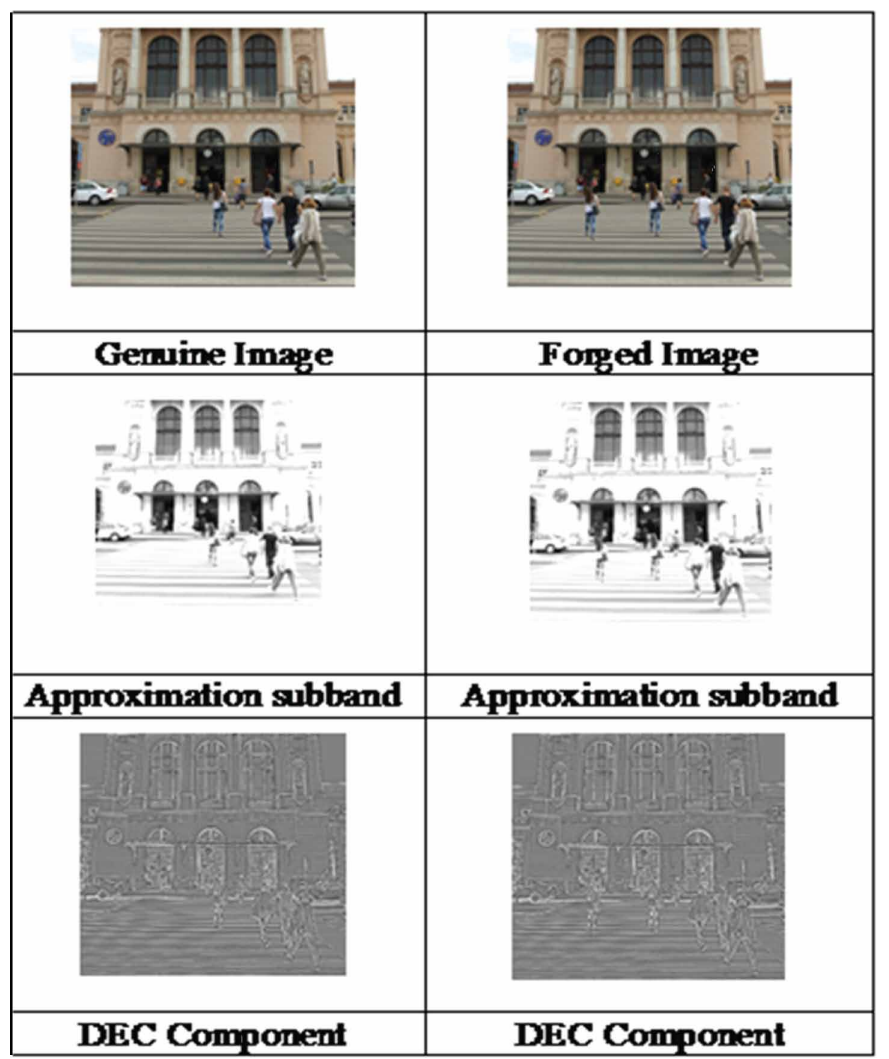

second set of experimentation concentrates on the performance against post-processing attacks. This investigation also evaluates the robustness of the three proposed methods against rotation and scaling attacks. Thirdly, a comparative analysis of the proposed methods with existing methods is performed.

\subsection{Image Dataset}

Experimentation is carried on public domain benchmark datasets CoMoFoD (Tralic et al., 2013) and CASIA ("CASIA Tampered Image Detection Evaluation Database,"). CoMoFoD database consists of 10,000 images of size $512 \times 512$ in .png format which are original, forged and post-processed images. CASIA Tampered Image Detection Evaluation Database consists of 800 authentic and 921 spliced color images of size $384 \times 256$ pixels with JPEG format.

\subsection{Classification}

In the proposed method, SVM (Vapnik, 2000) classifier with the polynomial kernel is used for classification of authentic and forged images. Quadratic programming is used to obtain the optimal parameters in the classification process. Texture feature vector obtained from a genuine image is labeled as +1 (positive), and CMF image as -1 (negative) label. SVM is a supervisory learning algorithm consists of train and test phases. In our experiments, feature vectors are randomly split into two sets as training and testing sets. SVM is modeled using the training set to define optimal hyperplane and the model is tested for classification using testing set. For better modeling, size of training set should be more than half. In the proposed methods, 7/8 of 400 images and 1/8 of 400 images are considered 
for training and testing sets respectively. The following parameters are calculated to evaluate the performance of the classifier:

True Positive (TP) - Forged Images predicted as Forged

True Negative (TN) - Original Images predicted as Original

False Positive (FP) - Original Images predicted as Forged

False Negative (FN) - Forged Images predicted as Original

With the above parameters, the below performance metrics are computed as defined in (6), (7) and (8):

$$
\text { Accuracy }=\frac{T P+T N}{T P+T N+F P+F N} \times 100
$$

Sensitivity $=\frac{T P}{T P+F N} \times 100$

$$
\text { Specificity }=\frac{T N}{T N+F P} \times 100
$$

\subsection{Performance of the Proposed Methods}

Proposed methods are validated using the performance metrics given in (6) to (8). Classification is performed using three proposed methods viz., 1. GLCM, 2. DWT-GLCM and 3. DEC-GLCM features. Table 2 shows the performance of the three proposed methods. Accuracy of DEC-GLCM

Table 2. Performance of the three proposed methods

\begin{tabular}{|l|l|l|l|}
\hline \multicolumn{1}{|c|}{ Proposed Method } & \multicolumn{1}{c|}{ Accuracy } & \multicolumn{1}{c|}{ Sensitivity } & \multicolumn{1}{c|}{ Specificity } \\
\hline DWT-GLCM & 90.75 & 86.5 & 95.5 \\
\hline GLCM & 92.25 & 88.5 & 96 \\
\hline DEC-GLCM & $\mathbf{9 5}$ & $\mathbf{9 1 . 5}$ & $\mathbf{9 6 . 5}$ \\
\hline
\end{tabular}

method is $95 \%$ and is almost 5\% higher when compared with DWT-GLCM and around 3\% in the case of GLCM only.

\subsection{Classifier Performance With Cross-Validation}

Images from the dataset are considered randomly for training and testing phases. This randomness affects the classifier accuracy. In order to reduce this effect, 10-fold Cross Validation (CV) is considered where the images in the dataset are split into 10 independent parts. In each CV test case, 9/10 of authentic and forged images are used for training the classifier and the rest 1/10 images are used for SVM classification. The average value of classifier performance for 10 -fold CV tests is considered as the final result. Figures 8-10 illustrates the performance parameters of the three proposed methods using 10-Fold Cross-validation. 
Figure 8. Accuracy of the three methods for 10-Fold CV

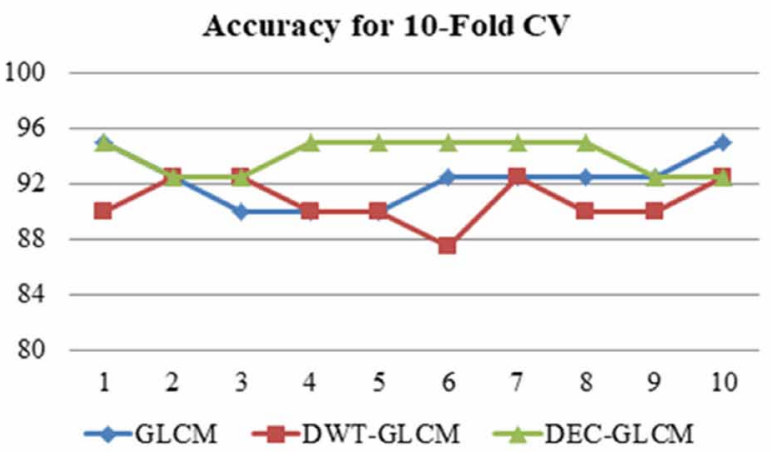

Figure 9 . Sensitivity of the three methods for 10 -Fold CV

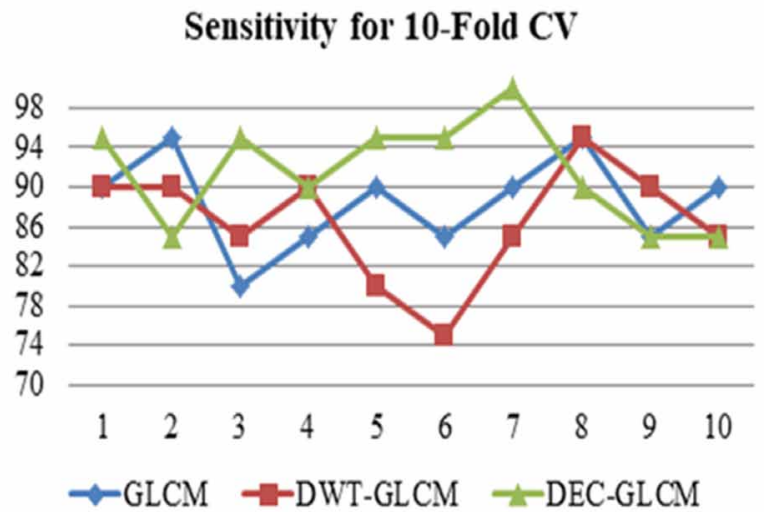

Figure 10 . Specificity of the three methods for 10 -Fold CV

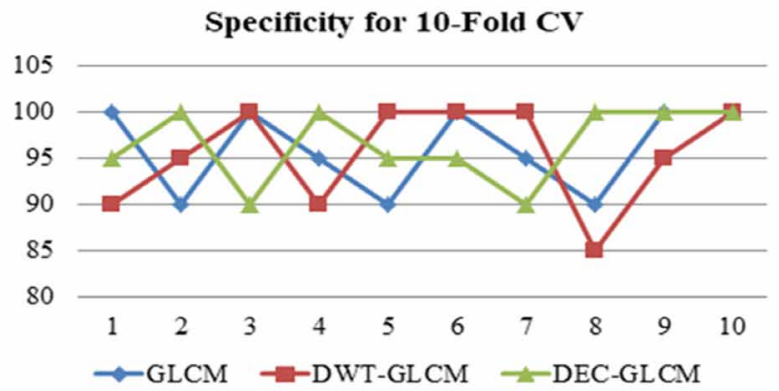




\subsection{Robustness Against Post-Processing Attacks}

The second set of experimentation is carried out to evaluate the performance of the three proposed methods against various post-processing attacks. Following are the post-processing attacks that are performed on the forged images:

1. Brightness Change (BC): Change in intensity level with lower and upper bounds $[(0.01,0.95)$, $(0.01,0.9),(0.01,0.8)]$;

2. Contrast Adjustments (CA): Contrast is varied with three different lower and upper bounds $[(0.01,0.95),(0.01,0.9),(0.01,0.8)]$;

3. Color Reduction (CR): Quantization is performed per each color channel as [32, 64, 128];

4. Noise Adding (NA): Additive White Gaussian Noise with mean, $\mu=0$, variance $\sigma^{2}=[0.009$, $0.005,0.0005]$;

5. Image Blurring (IB): Image is blurred with spatial averaging filter using [3x3, 5x5, 7x7] masks;

6. JPEG compression (JC): Images are compressed at different quality factors (Q) [20, 30, 40, $50,60,70,80,90,100]$.

The proposed method is evaluated by considering 50 forged images under each postprocessing attack category so that 1200 forged and processed images are tested. Figures 11-13 show the classification accuracy of the three proposed methods against various postprocessing attacks.

Figure 11. Accuracy against $B C, C A$ and $C R$ attacks

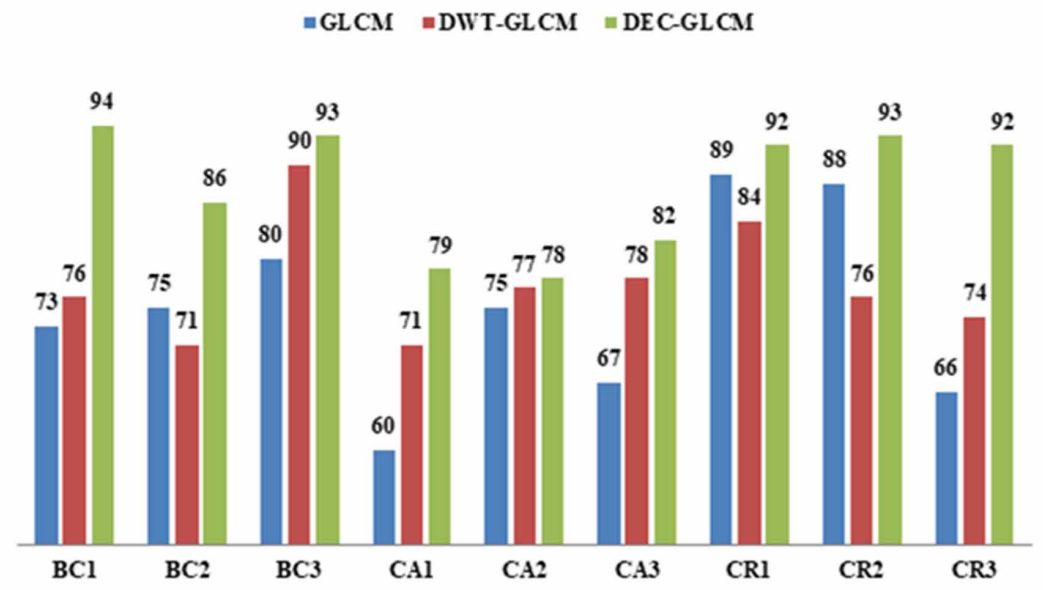

It is evident from Figure 11 that DEC-GLCM shows better robustness against BC, CA and CR attacks when compared with other methods viz., GLCM and DWT-GLCM.

It is apparent from Figure 12 that DEC-GLCM is unable to withstand the image blurring attack.

Average accuracy of 91.88 has been achieved for DEC-GLCM with JPEG quality factor ranging from 20 to 100. In general, quality factors above 40-50 are used. From Figure 13, it is evident that DEC-GLCM performs well in the presence of a JPEG compression attack. 


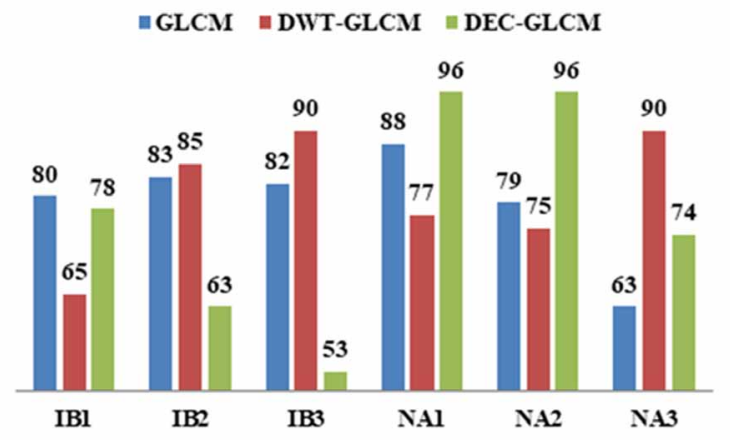

Figure 13. Accuracy against JPEG compression attacks for different quality factors

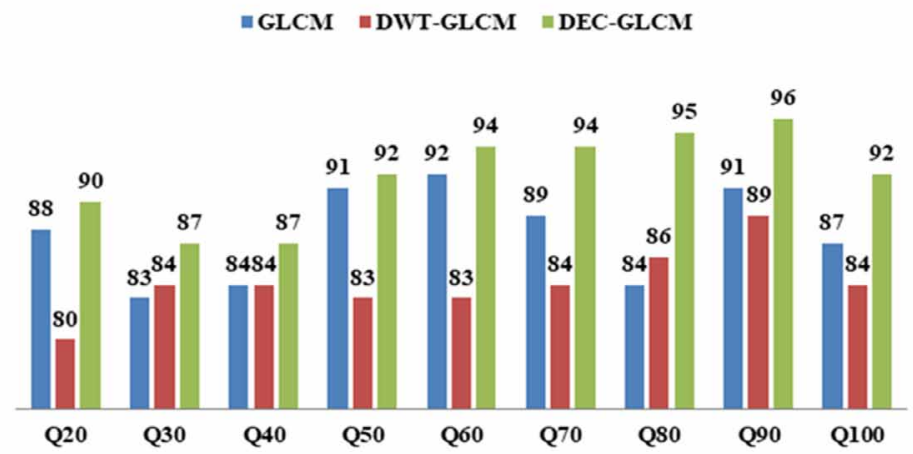

\subsection{Robustness Against Rotation and Scaling Attacks}

Robustness of the three proposed methods against rotation and scaling attacks on the forged regions are presented. The forged regions are rotated by some angle or scaled before it is pasted in the same image. Here, an effort is made to assess the robustness of the detection method against these attacks. Table 3 and Table 4 shows the detection accuracy against different rotation and scaling attacks respectively.

It is evident that all three proposed methods perform well against rotation and scaling attacks. DEC-GLCM out-performs the other two proposed methods.

\subsection{Comparative Analysis}

Comparative analysis of the three proposed methods with other existing methods in terms of detection accuracy and the feature size is presented in this section. Existing methods that are considered for comparison are SPT-LBP (Muhammad et al., 2014), Multi-WLD (Hussain et al., 2015), Multi-LBP (Hussain et al., 2015), LBP-DCT (Alahmadi et al., 2017), Multi-Texture (Vidyadharan \& Thampi, 2017) and BDCT (Prakash et al., 2018). All these methods are based on handcrafted features and work with the textural component in combination with an image transform for effective feature extraction. These existing methods are evaluated on CASIA v 1.0 dataset. Hence, the three proposed methods are evaluated using the same dataset on CMF images excluding spliced images for comparative analysis purpose. 
Table 3. Robustness against different rotation attacks

\begin{tabular}{|l|l|l|l|}
\hline \multicolumn{1}{|c|}{ Rotated Angle } & \multicolumn{1}{c|}{ DEC-GLCM } & \multicolumn{1}{c|}{ DWT-GLCM } & \multicolumn{1}{c|}{ GLCM } \\
\hline 2 & 93.05 & 88.88 & 87.77 \\
\hline 3 & 96.21 & 94.31 & 93.18 \\
\hline 4 & 97.17 & 96.5 & 95.87 \\
\hline 5 & 96.55 & 93.53 & 92.5 \\
\hline 7 & 97.22 & 94.9 & 93.2 \\
\hline 10 & 96.12 & 94.39 & 92.5 \\
\hline 40 & 99.53 & 98.61 & 97.23 \\
\hline 50 & 98.61 & 97.15 & 96.23 \\
\hline 90 & 97.68 & 96.75 & 95.43 \\
\hline 180 & 94.31 & 92.42 & 91.56 \\
\hline
\end{tabular}

Table 4. Robustness against different scaling factors

\begin{tabular}{|l|l|l|l|}
\hline \multicolumn{1}{|c|}{ Scaling Factor } & \multicolumn{1}{c|}{ DEC-GLCM } & \multicolumn{1}{c|}{ DWT-GLCM } & \multicolumn{1}{c|}{ GLCM } \\
\hline 0.40 & 98.61 & 97.69 & 96.77 \\
\hline 0.50 & 97.83 & 94.9 & 93.22 \\
\hline 0.60 & 96.15 & 94.25 & 92 \\
\hline 0.70 & 97.46 & 95.78 & 93.33 \\
\hline 0.80 & 97.68 & 96.22 & 95.57 \\
\hline 0.90 & 96.59 & 95.31 & 92.78 \\
\hline 0.95 & 97.87 & 95.68 & 93.61 \\
\hline 1.05 & 97.68 & 96.1 & 95.25 \\
\hline 1.10 & 97.29 & 96.75 & 95.75 \\
\hline 1.15 & 96.77 & 95.54 & 94.35 \\
\hline 1.30 & 94.82 & 93.67 & 92.82 \\
\hline
\end{tabular}

Comparative analysis of the three proposed methods with regard to feature size and detection accuracy is shown in Table 5. All three proposed methods work with a small feature vector of 24 dimensions. Proposed method-3 (DEC-GLCM) is superior to other existing methods in terms of detection accuracy.

As well, the proposed method-3 DEC-GLCM is compared with works based on deep learning techniques viz., (Rao \& Ni, 2016) and (Zhou et al., 2017). The two techniques use a feature vector of 400 and 576 respectively. They provide a detection accuracy of $97 \%$ approximately at the cost of high computational effort as given below in (9):

$$
O\left(\sum_{n=1}^{d}\left(n_{l-1} s_{l}^{2} n_{l} m_{l}^{2}\right)\right)
$$


Table 5. Comparative analysis of the three proposed methods with other existing methods

\begin{tabular}{|l|l|l|}
\hline \multicolumn{1}{|c|}{ Method } & \multicolumn{1}{c|}{ Feature Size } & \multicolumn{1}{c|}{ Accuracy } \\
\hline $\begin{array}{l}\text { SPT-LBP } \\
\text { (Muhammad et al., 2014) }\end{array}$ & 480 & 94.89 \\
\hline $\begin{array}{l}\text { Multi-WLD } \\
\text { (Hussain et al., 2015) }\end{array}$ & 770 & 91.25 \\
\hline $\begin{array}{l}\text { Multi-LBP } \\
\text { (Hussain et al., 2015) }\end{array}$ & 1203 & 85.56 \\
\hline $\begin{array}{l}\text { LBP-DCT } \\
\text { (Alahmadi et al., 2017) }\end{array}$ & - & 96 \\
\hline Multi-Texture (Vidyadharan \& Thampi, 2017) & 970 & 92.13 \\
\hline $\begin{array}{l}\text { BDCT } \\
\text { (Prakash et al., 2018) }\end{array}$ & 1944 & 87.5 \\
\hline Proposed Method (GLCM) & $\mathbf{2 4}$ & $\mathbf{9 0 . 4 5}$ \\
\hline $\begin{array}{l}\text { Proposed Method } \\
\text { (DWT-GLCM) }\end{array}$ & $\mathbf{2 4}$ & $\mathbf{9 2 . 6 8}$ \\
\hline $\begin{array}{l}\text { Proposed Method } \\
\text { (DEC-GLCM) }\end{array}$ & $\mathbf{2 4}$ & $\mathbf{9 6 . 5}$ \\
\hline
\end{tabular}

where, $l$ is the index of a convolutional layer, and $d$ is the depth (number of convolutional layers). $n_{l}$ is the number of filters (also known as "width") in the $l$-th layer. $n_{l-1}$ is also known as the number of input channels of the $l$-th layer. $s_{l}$ is the spatial size (length) of the filter. $m_{l}$ is the spatial size of the output feature map.

The detection process of the proposed method DEC-GLCM involves feature extraction and classification. Feature extraction involves time complexity of $O\left(n^{3}\right)$ and classification requires time of the order $O\left(n^{3}\right)$. Hence, the time complexity of the proposed method-3 DEC-GLCM is $O\left(n^{3}\right)$ and it achieved an accuracy of $96.5 \%$.

\section{CONCLUSION}

A novel approach for CMFD method is proposed using DEC image to build GLCM texture features. Texture features extracted from DEC-GLCM method are more distinguishable when compared to feature vectors obtained from DWT-GLCM and GLCM only. Classification efficacy of all the three proposed methods is assessed on CoMoFoD and CASIA v 1.0 datasets. The proposed methods work with a 24-dimension feature vector, exhibits resilience against post-processing attacks and is computationally effective. Evaluation of DEC-GLCM on CoMoFoD and CASIA yields an average accuracy of $95 \%$ and $96.5 \%$ respectively. Superiority of the proposed method DEC-GLCM is validated by comparison with existing methods viz., SPT-LBP (Muhammad et al., 2014), WLD-LBP (Hussain et al., 2015), LBP-DCT (Alahmadi et al., 2017), Multi-Texture (Vidyadharan \& Thampi, 2017) and BDCT (Prakash et al., 2018). The proposed methods concentrate on the detection of forged images, localization can also be carried out. 


\section{REFERENCES}

Agarwal, S., \& Chand, S. (2017). Image forgery detection using Markov features in undecimated wavelet transform. Proceedings of 9th International Conference on Contemporary Computing, IC3 2016 (pp. 1-6). AlHammadi, M. H., Muhammad, G., \& Hussain, M. B. G. (2013). Curvelet Transform and Local Texture Based Image Forgery Detection. In G. Bebis et al. (Eds.), Advances in Visual Computing. ISVC 2013 (pp. 503-512). Springer. doi:10.1007/978-3-642-41939-3_49

Al-Qershi, O. M., \& Khoo, B. E. (2013). Passive detection of copy-move forgery in digital images: State-of-theart. Forensic Science International, 231(1-3), 284-295. doi:10.1016/j.forsciint.2013.05.027 PMID:23890651

Alahmadi, A., Hussain, M., Aboalsamh, H., Muhammad, G., Bebis, G., \& Mathkour, H. (2017). Passive detection of image forgery using DCT and local binary pattern. Signal, Image and Video Processing, 11(1), 81-88. doi:10.1007/s11760-016-0899-0

Alhussein, M. (2016). Image tampering detection based on local texture descriptor and extreme learning machine. Proceedings of 2016 UKSim-AMSS 18th International Conference on Computer Modelling and Simulation, UKSim (pp.196-199). doi:10.1109/UKSim.2016.39

CASIA Tampered Image Detection Evaluation Database. (2013). Retrieved from http://forensics.idealtest.org/ casiav1/

Chen, J., Shan, S., He, C., Zhao, G., Pietikainen, M., Chen, X., \& Gao, W. (2009). WLD: A robust local image descriptor. IEEE Transactions on Pattern Analysis and Machine Intelligence, 32(9), 1705-1720.

Dixit, R., \& Naskar, R. (2017). Review, analysis and parameterisation of techniques for copy-move forgery detection in digital images. IET Image Processing, 11(9), 746-759. doi:10.1049/iet-ipr.2016.0322

Fridrich, J., Soukal, D., \& Lukáš, J. (2003). Detection of Copy-Move Forgery in Digital Images. Proceedings of Digital Forensic Research Workshop (pp.19-23). doi:10.1109/PACIIA.2008.240

Haralick, R. M., Shanmugam, K., \& Dinstein, I. (1973). Textural Features for Image Classification. IEEE Transactions on Systems, Man, and Cybernetics. SMC, 3(6), 610-621. doi:10.1109/TSMC.1973.4309314

Hashmi, M. F., \& Keskar, A. G. (2015). Block and fuzzy techniques based forensic tool for detection and classification of image forgery. Journal of Electrical Engineering \& Technology, 10(4), 1886-1898. doi:10.5370/ JEET.2015.10.4.1886

Hashmir, M. F., \& Keskar, A. G. (2015). Image Forgery Detection and Classification using HMM and SVM Classifier. International Journal of Security and Its Applications, 9(4), 125-140. doi:10.14257/ijsia.2015.9.4.13

Hussain, M., Muhammad, G., Saleh, S. Q., Mirza, A. M., \& Bebis, G. (2012). Copy-Move Image Forgery Detection Using Multi-Resolution Weber Descriptors. Proceedings of Eighth International Conference on Signal Image Technology and Internet Based Systems (pp. 395-401). Academic Press. doi:10.1109/SITIS.2012.64

Hussain, M., Qasem, S., Bebis, G., Muhammad, G., Aboalsamh, H., \& Mathkour, H. (2015). Evaluation of Image Forgery Detection Using Multi-Scale Weber Local Descriptors. International Journal of Artificial Intelligence Tools, 24(4), 1-28. doi:10.1142/s0218213015400163

Isaac, M. M., \& Wilscy, M. (2015). Image Forgery Detection Based on Gabor Wavelets and Local Phase Quantization. Proceedings of Procedia Computer Science, 58, 76-83. doi:10.1016/j.procs.2015.08.016

Li, C. (Ed.). (2013). Emerging Digital Forensics Applications for Crime Detection, Prevention, and Security. IGI-Global; doi:10.4018/978-1-4666-4006-1

Mahdian, B., \& Saic, S. (2010). A bibliography on blind methods for identifying image forgery. Signal Processing Image Communication, 25(6), 389-399. doi:10.1016/j.image.2010.05.003

Mangat, S. S., \& Kaur, H. (2016). Improved copy-move forgery detection in image by feature extraction with KPCA and adaptive method. Proceedings of 2nd International Conference on Next Generation Computing Technologies NGCT 2016 (pp. 694-703). Academic Press. doi:10.1109/NGCT.2016.7877501

Muhammad, G., Al-Hammadi, M. H., Hussain, M., \& Bebis, G. (2014). Image forgery detection using steerable pyramid transform and local binary pattern. Machine Vision and Applications, 25(4), 985-995. doi:10.1007/ s00138-013-0547-4 
Muhammad, G., Al-Hammadi, M. H., Hussain, M., Mirza, A. M., \& Bebis, G. (2013). Copy move image forgery detection method using steerable pyramid transform and texture descriptor. Proceedings of IEEE EuroCon, 2013, 1586-1592. doi:10.1109/EUROCON.2013.6625188

Popescu, A. C., \& Farid, H. (2004). Exposing digital forgeries by detecting duplicated image regions. Dartmouth College. doi:10.1109/TSP.2004.839932

Prakash, C. S., Kumar, A., Maheshkar, S., \& Maheshkar, V. (2018). An integrated method of copy-move and splicing for image forgery detection. Multimedia Tools and Applications, 1-25. doi:10.1007/s1 1042-018-5899-3

Rao, Y., \& Ni, J. (2016). A Deep Learning Approach to Detection of Splicing and Copy-Move Forgeries in Images. Proceedings of IEEE International Workshop on Information Forensics and Security (WIFS) (pp. 1-6). IEEE Press. doi:10.1109/WIFS.2016.7823911

Saurabh, Agarwal., \& Satish, Chand. (2015). Image Forgery Detection using Multi Scale Entropy Filter and Local Phase Quantization. International Journal of Image. Graphics and Signal Processing, 8(10), 78-85. doi:10.5815/ijigsp.2015.10.08

Sekhar, R., \& Shaji, R. S. (2014). A Methodological Review on Copy-Move Forgery Detection for Image Forensics. International Journal of Digital Crime and Forensics, 6(December), 34-49. doi:10.4018/ijdcf.2014100103

Shen, X., Shi, Z., \& Chen, H. (2017). Splicing image forgery detection using textural features based on the grey level co-occurrence matrices. IET Image Processing, 11(1), 44-53. doi:10.1049/iet-ipr.2016.0238

Suresh, G., \& Srinivasa Rao, Ch. (2016). Copy Move Forgery Detection Using GLCM Based Statistical Features. International Journal on Cybernetics \& Informatics, 5(4), 165-171. doi:10.5121/ijci.2016.5419

Tralic, D., Zupancic, I., Grgic, S., \& Grgic, M. (2013). CoMoFoD - New Database for Copy-Move Forgery Detection. Proceedings of 55th International Symposium ELMAR-2013. Academic Press.

Vapnik, V. N. (2000). The Nature of Statistical Learning Theory (Vol. 8). Springer. doi:10.1007/978-1-47573264-1

Vidyadharan, D. S., \& Thampi, S. M. (2017). Digital image forgery detection using compact multi-texture representation. Journal of Intelligent \& Fuzzy Systems, 32(4), 3177-3188. doi:10.3233/JIFS-169261

Wu, M. L., Fahn, C. S., \& Chen, Y. F. (2017). Image-format-independent tampered image detection based on overlapping concurrent directional patterns and neural networks. Applied Intelligence, 47(2), 347-361. doi:10.1007/s10489-017-0893-4

Yang, B., Sun, X., Chen, X., Zhang, J., \& Li, X. (2013). An efficient forensic method for copy-move forgery detection based on DWT-FWHT. Wuxiandian Gongcheng, 22(4), 1098-1105.

Zhang, X., Cui, J., Wang, W., \& Lin, C. (2017). A study for texture feature extraction of high-resolution satellite images based on a direction measure and gray level co-occurrence matrix fusion algorithm. Sensors (Switzerland), 17(7), 1-15. doi:10.3390/s17071474 PMID:28640181

Zhou, J., Ni, J., \& Rao, Y. (2017, August). Block-based convolutional neural network for image forgery detection. In International Workshop on Digital Watermarking (pp. 65-76). Cham: Springer. doi:10.1007/978-3-319-64185-0

Gulivindala Suresh is a research scholar in the Department of ECE at JNTU-K University College of Engineering, AP, India. He obtained his M. Tech from Biju Patnaik University of Technology, Orissa, India. He obtained his B. Tech from JNTU, AP, India. His research interests are digital image processing and VLSI. He is a member of IETE. Presently, he is working as Assistant Professor in the Department of ECE, GMR Institute of technology, Rajam, AP, India.

Srinivasa Rao Ch is currently working as a Professor in the Department of ECE, JNTUK University College of Engineering, Vizianagaram, AP, India. He obtained his Ph.D. in the Digital Image Processing area from the University College of Engineering, JNTUK, Kakinada, AP, India. He received his $M$. Tech degree from the same institute. He published more than 50 research papers in international journals and conferences. He is a reviewer for many reputed international journals. His research interests are digital speech/image and video processing and evolutionary algorithms. He is a member of the IEEE. Dr. Rao is a Fellow of IETE and Member of CSI. 